\title{
Surgineering: a new type of collaboration among surgeons and engineers
}

\author{
H. Feussner ${ }^{1}$ - D. Wilhelm ${ }^{1} \cdot$ N. Navab ${ }^{2}$ - A. Knoll ${ }^{3} \cdot$ T. Lüth ${ }^{4}$
}

Received: 6 September 2018 / Accepted: 28 November 2018 / Published online: 11 December 2018 (c) CARS 2018

Today, progress in surgery is mainly driven by technological advances. Accordingly, technological research and development gain a growing influence on all medical interventional disciplines. In the past, this has seemingly been different, since many minor and major breakthroughs were initiated by technically inspired surgeons (hypobaric chamber or suture staplers, just to name a few). Today, however, this has changed.

Biomedical engineering issues as well as their technical solutions have become too sophisticated and specialized for today's surgeons to significantly contribute to new technical solutions in detail. Too often, the role of the surgeon is limited to accompany the development process, or more frequently, to evaluate a product already commercially available.

Nonetheless, the surgical partnership is still fundamental in biomedical engineering, starting with the very beginning of a new project. Only the surgeon himself, as the user, is able to define the medical problem or to identify a deficit in surgical practice that is supposed to be overcome by a new technical development. As soon as the engineer has come to a first solution or is able to provide a first prototype, the surgeon is called up again to evaluate the suitability and may contribute fresh ideas for its improvement.

$凶$ H. Feussner

hubertus.feussner@tum.de

1 Department of General and Visceral Surgery, Klinikum rechts der Isar Munich, Technical University of Munich, Munich, Germany

2 Chair for Computer Aided Medical Procedures and Augmented Reality, Institute of Informatic I16, Technische Universität München, Boltzmannstr. 3, 85748 Garching bei München, Germany

3 Chair of Robotics and Embedded Systems, Institute of Informatic VI, Technische Universität München, Boltzmannstraße 3, 85748 Garching bei München, Germany

4 Chair of Micro Technology and Medical Device Technology, TUM Department of Mechanical Engineering, Technische Universität München, Boltzmannstraße 3, 85748 Garching bei München, Germany
Finally, it is a surgical task to prepare and to perform preclinical and clinical trials to find out whether the new product/solution is mature for practical medical care and to confirm or rule out its superiority over conventional solutions and current medical standards.

This typical modern role of a surgeon is, to some extent, comparable to that of a race driver. The task of a formula 1 pilot is to use the car in the best way in order to gain the victory. Of course, he may also give some valuable practical advice, but design and construction of the bolide is the domain of the development team.

At our institutions, an interdisciplinary team of surgeons and engineers looks back at many years of successful collaboration. During these years, a particular phenomenon of cooperation evolved which is beyond the scope of the classical distribution of roles as described above.

The classical separation between surgical and engineering tasks was substituted in some instances by interactive steps. It happened occasionally that some parts of a surgical intervention were absolutely impossible to realize from the engineering viewpoint (Fig. 1).

In some of these cases, however, the team was yet unable to overcome the barrier: a developmental cul-de-sac. This is a situation when current technology comes to its limits and further developmental efforts are completely unpromising. The surgeons thought about how the same surgical goal could be reached by another procedural approach, by modifying some aspects of the surgical manipulation in a way which made it easier to be handled by a technical approach. Of course, this modification must not compromise the final aim of the operation, but it was striking how frequently various ways could be detected to reach the same goal. This variation of the surgical tactics offered a new chance for the engineer to design the required instruments. (Fig. 2)

We coined the term "surgineering" as a neologism to describe this new type of a collaboration [1]. It is an abbreviated combination of Surgery and Engineering. Surgineering results in more flexibility and allows for a modification of the original requirement and target specifications. It opens 
Fig. 1 Developmental cul-de-sac: Sometimes it is impossible to overcome the technical problems of a particular part of the surgical intervention by any conceivable technical approach

\section{Problem}

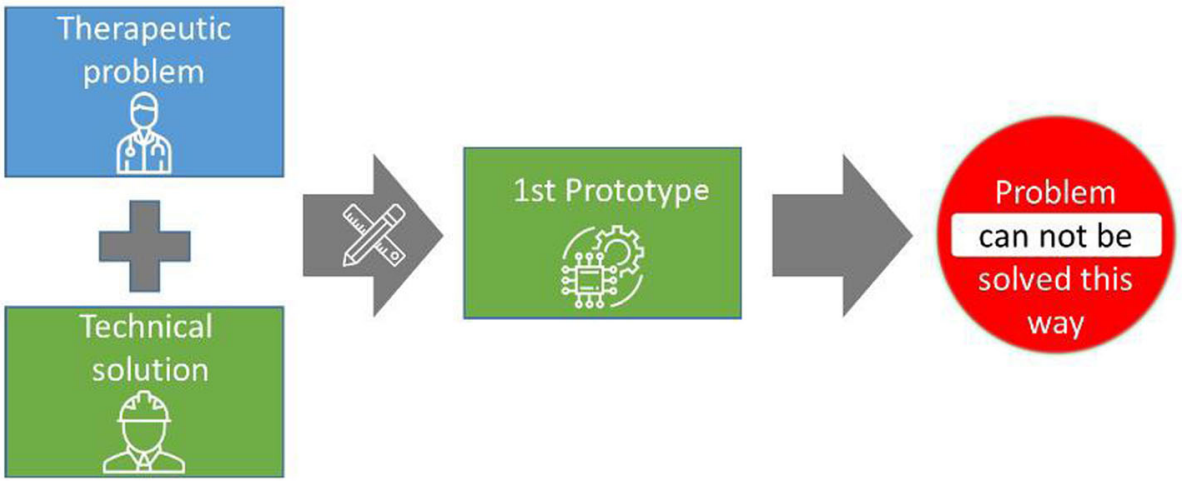

\section{Problem}

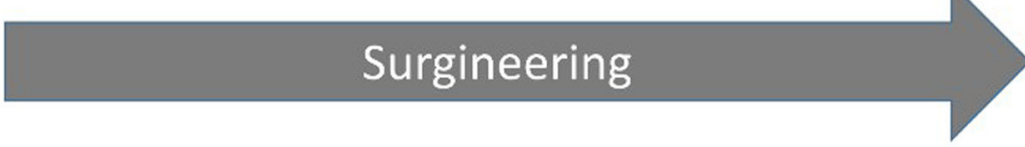

\section{Solution}
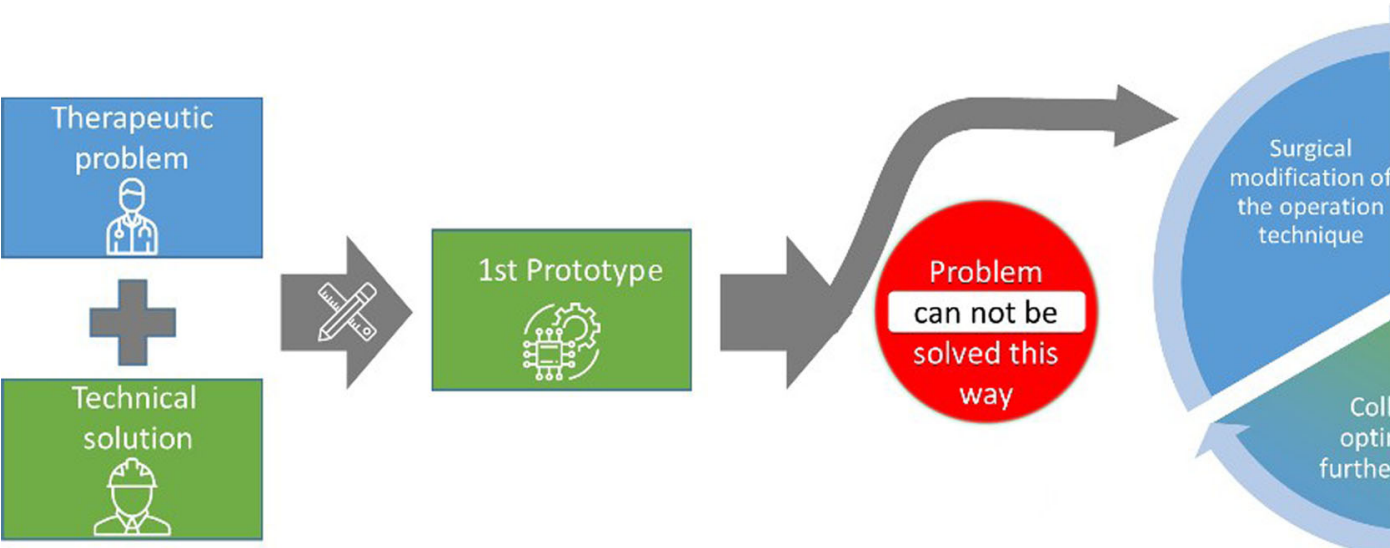

the option of

the operation
technique

Technical

modifications

$+$

upgrades

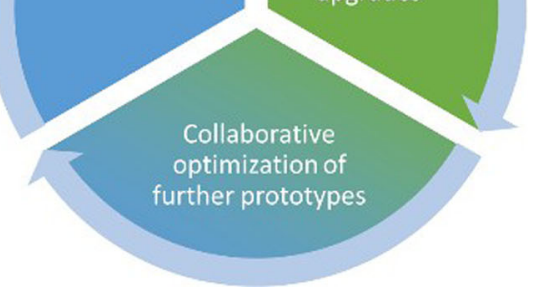

Fig. 2 If, for a particular step of the operation, a technical solution appears to be inconceivable, a (slight) modification of the surgical procedure might open new doors

the floor to real and substantial innovation and to groundbreaking changes revisions of our daily surgical work.

A practical application of surgineering may be illustrated by the development of a new type of single-port sigmoid resection. With a first prototype of a telemanipulation system ("platform"), we were able to do relatively simple surgical operations such as the removal of the appendix or gallbladder. More complex operations such as colonic resections, however, were impossible to perform. Though many steps of a standard sigmoid resection could be realized, resection of the respective colonic segment and specimen removal proved to be unsurmountable hurdles.

The breakthrough could be achieved by the decision of the surgeons to use the rectal stump as an auxiliary access for resection and specimen retrieval. This led to the first successful prototype as a proof of concept. Of course, several more optimization loops followed (Fig. 3).
They were mainly of a technical nature, but one of them also included another "surgineering" step. In our single-port sigmoid resection, the distal stump of the rectum has to be perforated by the pin of the stapler. This is facilitated by a plastic mandrel which is temporarily mounted to the tip of the pin. As soon as the perforation is achieved, the mandrel has to be removed to unite the anvil with the pin of the stapler. This, however, was extremely difficult when using the new platform since it was very difficult to grasp the plastic mandrel with the rather small jaws of the grasping forceps. If we succeeded, the force was often too low to extract the plastic part out of the pin.

The problem was solved by a modification of the operation technique. The surgeon no longer closed the pursue string before the insertion of the stapler but only after the advancement of the pin into the abdominal cavity. Thus, the 


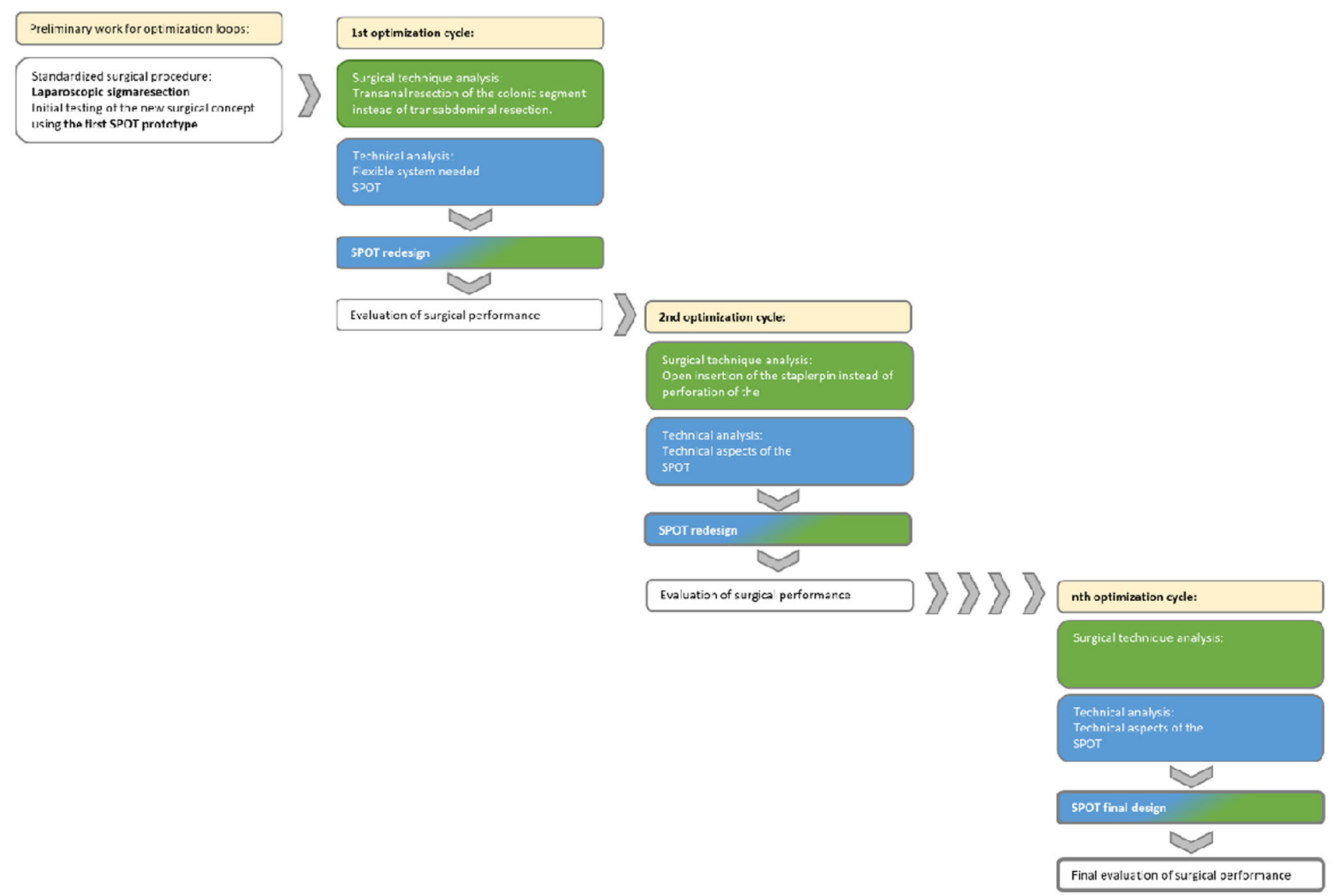

Fig. 3 Iterative development process. After an analysis of the initial state, several optimization cycles are performed. An analysis of technical aspects and surgical procedures is carried out in each cycle. These analyses result in modifications both in technical and surgical regards and thus form a new basis for the next operation cycle
Fig. 4 a Normally, the occluded rectal stump is perforated by the pin of the stapler. The perforation is enabled by a plastic mandrel, which, afterward has to be removed to unite the stapler with the anvil. The removal of the mandrel is very difficult if not impossible using the SPOT or HVSPS. b Leaving the pursue string suture open enables easy introduction of the pin without a mandrel

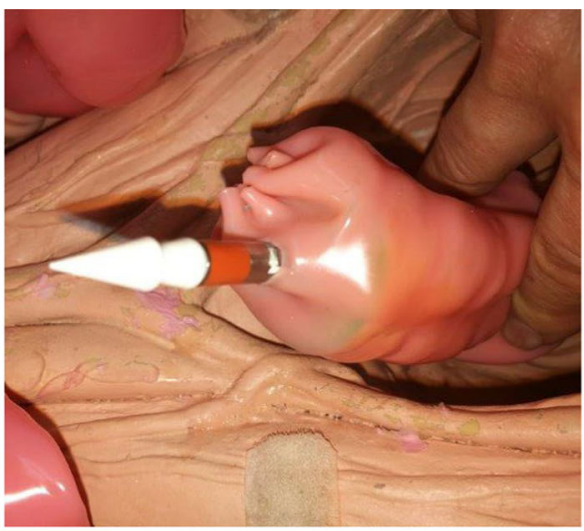

(a)

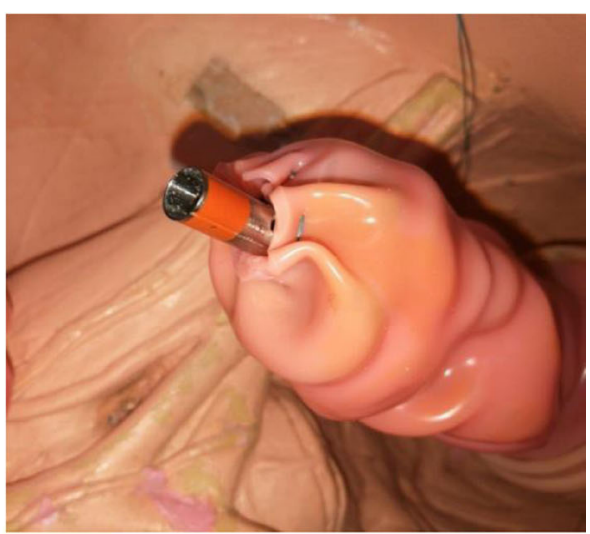

(b) mandrel was no longer necessary, and an important element of retardation could be eliminated (Fig. 4).

Surgineering is an iterative process model during the development process. After an analysis of the initial state, several optimization cycles are performed. An analysis of both technical aspects and surgical procedures is carried out in each cycle. These analyses result in modifications and thus form the basis of the next operation cycle.

"Surgineering" is not confined to mechanical engineering. Another example may be given from computer science. A part of our scientific activities is the reconstruction of the surgical workflow. A suitable model is the removal of the 
Fig. 5 Different ways to occlude and to dissect in Calot's triangle: a conventional sequence: First, both the cystic artery and the cystic duct are occluded by clips (at random). If the last clip is fired, both structures are cut by the scissors. b "Surgineering" approach: The cystic artery is ligated first. After three clips, it is dissected as well. The type of "substructing" of a surgical step makes it more suitable for easy identification
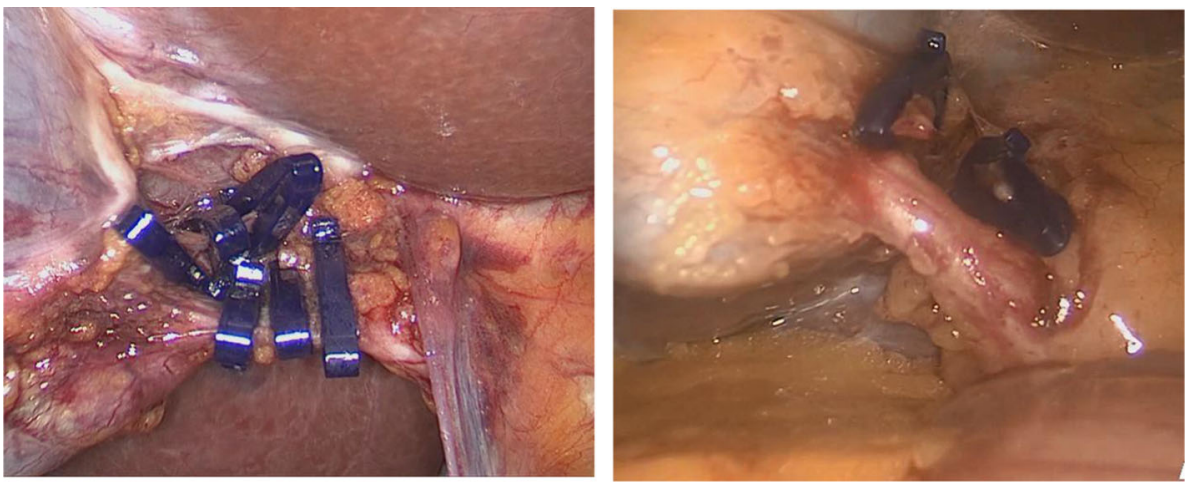

(a) (b) gallbladder. One part is to separate the distinct phases of the operation from each another. This can be done by identifying the sequence of instruments. In our work, one phase was very difficult to detect: the part of the operation when the cystic duct and the cystic artery were occluded by clips and severed. Originally, six clips were applied $(3 \times$ cystic artery and $3 \times$ cystic duct), and then, both tubular structures were dissected by the scissors.

A simple modification of the procedure made it by far easier to the system to understand what was going on. We no longer waited for the positioning of all clips, but decided to clip and dissect the cystic artery first and then continue with the cystic duct. This new sequence made this step of the operation by far more characteristic and unambiguous (Fig. 5).

Surgery is a very traditional discipline, and most surgeons are educated and trained to reproduce and apply exactly the same surgical techniques as their proctors. Distinct parts of the same operation may vary from hospital to hospital, since evidence-based and obligatory common standards are rare. Modifications are avoided. Only a handful of innovators are open to the integration of new principles and technologies into their work. This is a main barrier for a fast transformation of surgery. With the aim of improving upon today's level of surgery, surgeons should open their minds to innovative technical approaches. If they comprehend at least a spark of improvement and the chance to revolutionize surgery, they should be ready to modify traditional approaches if necessary.
We are firmly convinced that the principle of our approach-bilateral flexibility both on the sides of the engineers and the surgeons - is already practiced in many biomedical engineering workgroups all over the world, but to the best of our knowledge, it has never been defined explicitly. The dissemination of this idea of "surgineering" could hopefully promote the concept of translational research and shorten the way from "bench to bedside." CARS has always been the herald of interdisciplinary collaboration among clinicians and engineers/computer scientists/basic research and, therefore, should be the natural protagonist of the idea of "surgineering" [2].

Acknowledgements This work was supported in part by DF6.

\section{Compliance with ethical standards}

Conflict of interest The authors declare that they have no conflict of interest.

\section{References}

1. Feussner $\mathrm{H}$ The role of technology in minimally invasive surgery: "Surgineering" Department of Surgery/Research Group Minimally invasive Interdisciplinary Therapy. https://www.nct-heidelberg.de/ fileadmin/media/das_nct/. Accessed 1 July 2017

2. Lemke HU (2017) Editorial for clinical investigations: toward the vision and spirit of CARS with innovative clinical investigations. Int J CARS. https://doi.org/10.1007/s11548-01-1586-5 